\title{
Energy-Efficient Communication over the Unsynchronized Gaussian Diamond Network
}

\author{
Ritesh Kolte, Urs Niesen, and Piyush Gupta
}

\begin{abstract}
Communication networks are often designed and analyzed assuming tight synchronization among nodes. However, in applications that require communication in the energy-efficient regime of low signal-to-noise ratios, establishing tight synchronization among nodes in the network can result in a significant energy overhead. Motivated by a recent result showing that near-optimal energy efficiency can be achieved over the AWGN channel without requiring tight synchronization, we consider the question of whether the potential gains of cooperative communication can be achieved in the absence of synchronization. We focus on the symmetric Gaussian diamond network and establish that cooperative-communication gains are indeed feasible even with unsynchronized nodes. More precisely, we show that the capacity per unit energy of the unsynchronized symmetric Gaussian diamond network is within a constant factor of the capacity per unit energy of the corresponding synchronized network. To this end, we propose a distributed relaying scheme that does not require tight synchronization but nevertheless achieves most of the energy gains of coherent combining.
\end{abstract}

\section{INTRODUCTION}

\section{A. Motivation}

An often implicit assumption in the design and analysis of communication networks is that the clocks of different nodes in the network are tightly synchronized. In practice, near-tight synchronization is achieved via periodic transmission of pilot signals or via differential modulation of data bits. These strategies result in little energy overhead in the high-SNR regime, thus justifying the assumption of tight synchronization. However, in applications such as space communication or wireless sensor networks where energy efficiency is of critical importance, communication has to take place in the low-SNR regime. In this regime, the energy overhead incurred in establishing near-tight synchronization using the aforementioned approaches can be significant, resulting in suboptimal energy efficiency.

Recently, [1] has established a fundamental limit on the optimal energy efficiency, measured in bits per unit energy, that can be achieved over the point-to-point AWGN channel without synchronization between the transmitter and the receiver. This limit is nearly equal to the optimal energy efficiency of the perfectly synchronized channel, and, in contrast to the conventional approach, the corresponding achievable scheme does not attempt to tightly synchronize clocks.

Motivated by this result, we ask whether near-optimal energy efficiency can be achieved in a distributed cooperative communication setting where the participating nodes may not be synchronized. Multiple cooperating nodes can potentially offer significant improvements in the communication rate and energy efficiency by providing power and multiplexing gains. However it is widely believed that establishing tight synchronization is essential to achieve these gains.

As an example, consider a symmetric Gaussian multiple-access channel where $K$ transmitters have a common message that is to be delivered to the destination. With perfect synchronization, the transmitting nodes can perform beamforming (i.e., achieve coherent combining of their transmitted signals at the destination) thereby communicating $K$ times more bits per unit energy than over a point-to-point channel. However, if the nodes are not tightly synchronized, coherent combining of the transmitted signals at

R. Kolte is with the Department of Electrical Engineering at Stanford University. U. Niesen and P. Gupta are with Bell Labs, Alcatel-Lucent. Emails: rkolte@stanford.edu, urs.niesen@alcatel-lucent.com, piyush.gupta@alcatel-lucent.com

This work was presented at the IEEE International Symposium on Information Theory 2014 in Honolulu, HI, USA.

This work was supported in part by AFOSR under grant FA9550-09-1-0317. 
the destination is not guaranteed. On the other hand, the performance of conventional schemes that first establish tight synchronization can also be suboptimal [1]. This poses the question if these cooperative communication gains are still achievable in the absence of node synchronization.

\section{B. Summary of Results}

In this paper, we consider the problem of communicating over an unsynchronized symmetric $K$-relay Gaussian diamond network. To focus on communicating in the energy-efficient regime, we adopt capacity per unit energy as the performance metric instead of the usual notion of channel capacity. Our main result is that the potential gains in energy efficiency due to the presence of multiple relays are achievable up to a constant multiplicative factor even in the absence of synchronization, where the constant is independent of the channel gains and the number of relays.

To establish this result, we propose a distributed communication scheme that achieves energy-efficient forwarding at the relays and coherent combining of the signals at the destination by compensating for synchronization errors. This is done by concatenating an outer random code with an inner repetition code. The inner code provides robustness against the lack of synchronization and its length and scaling factor are chosen so that every outer-code symbol can be transmitted reliably with minimum energy. The power levels of the outer random code at the source and at the relays are chosen depending on the relative values of the first-hop gain, the second-hop gain, and the number of relays in order to obtain near-optimal energy efficiency.

Our model of the unsynchronized symmetric Gaussian diamond network is depicted in Fig. 1(b) for $K=2$ relays. The unsynchronized network is obtained by introducing independent insertion/deletion channels on all channels in the standard (synchronized) symmetric Gaussian diamond network, depicted in Fig. 1(a), The approach of modeling synchronization errors via insertions/deletions is motivated by noting that if the clock of a receiving node exhibits drift and jitter with respect to the clock at the transmitting node, then the receiver may sample the transmitted signal either faster than the transmitter, leading to insertions, or slower, leading to deletions.

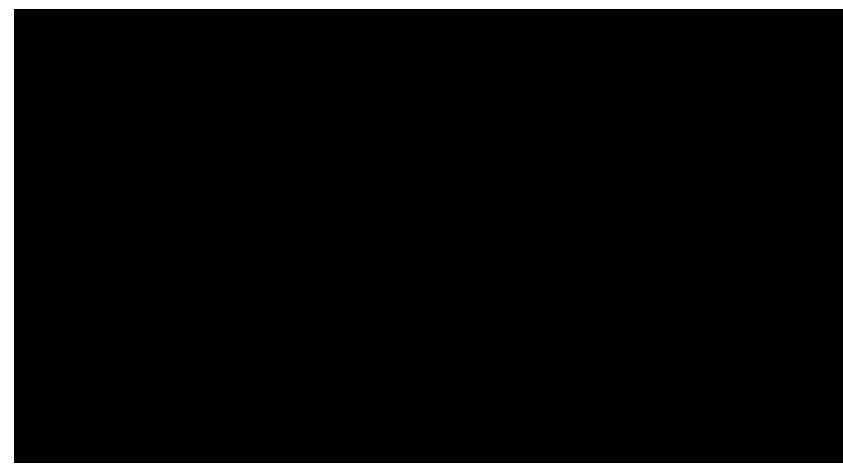

(a)

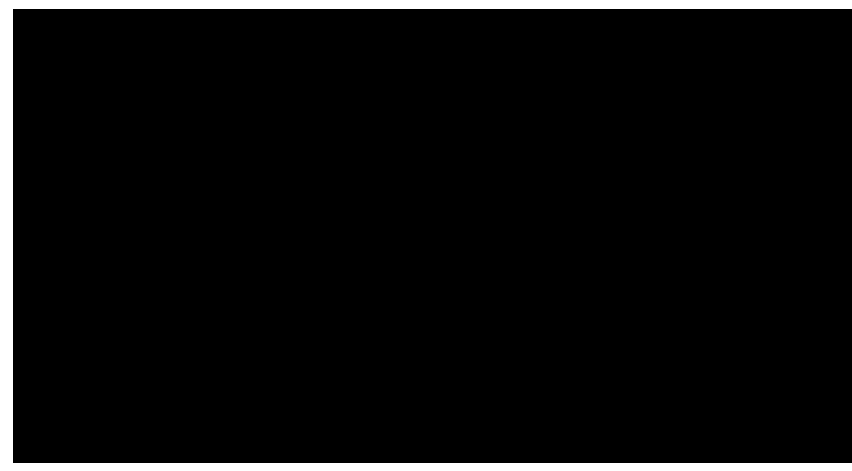

(b)

Fig. 1. Synchronized (a) and unsynchronized (b) two-relay Gaussian diamond networks.

\section{Related Work}

The insertion/deletion channel as a model for communication with synchronization errors was introduced by Dobrushin in [2]. Finding the capacity of this channel is still an open problem, the main difficulty being the channel memory introduced by the insertions and deletions. As a result of the difficulty in analyzing the general insertion/deletion channel, most of the literature focuses on a simpler special case, the binary deletion channel, for which good approximations have been developed. However, the exact capacity for this special case too remains elusive. We refer the reader to [3] and references therein for a survey on this topic up to 2009. Some of the work that has appeared since 2009 is [4], [5], [6], [7], [8]. 
The notion of capacity per unit energy was analyzed for the synchronized AWGN channel in [9]. It is shown there that pulse-position modulation achieves the capacity per unit energy of this channel. Singleletter characterizations for the capacity per unit cost for general cost functions and general synchronized discrete memoryless channels were found in [10]. However, these results depend on the channel being memoryless, whereas the channel considered here has memory due to the presence of insertions and deletions. Hence, these results cannot be directly applied here. The pulse-position modulation scheme was generalized for unsynchronized channels in [1], where it was shown to achieve the capacity per unit energy of the unsynchronized AWGN channel. We point out that, pulse-position modulation itself cannot be extended to the unsynchronized diamond network considered in this paper. Indeed, unless the relays decode the message sent by the transmitter, they end up spending a significant amount of energy forwarding noise. However, requiring the relays to decode the message can also be suboptimal—for instance, when the source-relay channel is weak.

Since its introduction in [11], there have been numerous works analyzing the capacity of the synchronized Gaussian diamond network. The achievable rates of two well-known relaying schemes, decodeforward and amplify-forward, were analyzed for the two-relay diamond network in [11]. To counter the poor performance of amplify-forward at low SNR, the bursty amplify-forward scheme was proposed in [12]. This scheme was shown to be approximately optimal for the symmetric Gaussian diamond network with arbitrary number $K$ of relays in [13], both in the sense of a uniform additive gap of 1.8 bits and a uniform multiplicative gap of a factor 14 .

The constant multiplicative approximation guarantee provided in [13] implies that bursty amplify-andforward also achieves the capacity of the synchronized symmetric Gaussian diamond per unit energy up to the same multiplicative gap. For the symmetric diamond networks with only two relays, it was shown in [14] that bursty amplify-forward and superposition-partial-decode-forward achieve the capacity per unit energy up to a factor of at most 2.85 and at most 1.87 , respectively. The capacity per unit energy of the canonical synchronized single-relay channel was approximated to within a factor 1.7 in [15]. A bursty amplify-and-forward scheme was also shown to achieve the optimal outage capacity per unit energy for the frequency-division relay channel at low SNR and at low outage probability [16]. It is worth pointing out that the bursty constructions mentioned here do not generalize to the unsynchronized diamond network considered in this paper, since, due to the synchronization errors, the bursty codewords cannot be made to combine constructively at the destination.

Different types of synchronization errors have been considered in the literature. Insertion/deletion channels as used in this paper model clock drift and jitter at the symbol level. A different approach is to assume that the clocks at different nodes only differ by a constant offset with respect to each other. For such errors, the offset is typically either assumed to be equal to a multiple of the symbol interval (frame asynchronism [17]) or equal to a length less than one symbol interval (symbol asynchronism). The multiple-access channel capacity region under symbol asynchronism and frame asynchronism has been analyzed in [18] and [19], [20], respectively. Several recent works, [21] and [22], address the energy efficiency of bursty data communication. Here, asynchronism is not in the sense of symbol or frame asynchronism as described above, but in the sense that data arrives at the source sporadically at some random time unknown beforehand.

Another model for asynchronism has been proposed and analyzed in [23]. Here, the asynchronism is modeled by stretching or shrinking the continuous-time transmitted signal by a time-dependent factor. This model can be thought of as the continuous-time analog of the asynchronism model that we use, however a number of additional assumptions are required to make the continuous-time problem mathematically tractable: the compress/stretch factor is assumed to lie between two positive numbers (i.e. bounded), the communication system is assumed to be noiseless and have infinite bandwidth, the allowed run-lengths lie in a closed positive interval and the input alphabet is finite. 


\section{Organization}

We describe the problem setting formally in Section [II. The main results are presented in Section III The proof of these results are contained in Sections IV and V.

\section{SYSTEM MODEL}

We first describe the behavior of the stand-alone insertion/deletion channel (IDC) in Section II-A. The model of the overall diamond network is described in Section II-B.

\section{A. Insertion/Deletion Channel}

Let $(x[1], \ldots, x[T]) \in \mathcal{X}^{T}$ denote the input to the IDC. The actions of the IDC are governed by an i.i.d. sequence of states $(s[1], \ldots, s[T]) \in\{0,1,2, \ldots\}^{T}$. The state random variable $s[t]$ describes how many times input symbol $x[t]$ appears at the output of the IDC. Let $(\tilde{x}[1], \ldots, \tilde{x}[\tilde{L}]) \in \mathcal{X}^{\tilde{L}}$ denote the output of the IDC, where $\tilde{L}$ is the random output length and is equal to $\sum_{t=1}^{T} s[t]$. We refer the reader to [1, Example 1] for an example of the operation of the IDC. We denote by

$$
\mu \triangleq \mathbb{E}(s[1])
$$

and

$$
\sigma^{2} \triangleq \operatorname{var}(s[1])
$$

the mean and the variance of the insertion/deletion process, respectively, and we refer to any IDC with those parameters as $\operatorname{IDC}\left(\mu, \sigma^{2}\right)$. Here, $\mu$ and $\sigma^{2}$ can be interpreted as capturing the drift and jitter of the receiver clock, respectively. As noted in [1], in practice the parameter $\mu$ is close to 1 , e.g., $\mu=1 \pm 10^{-4}$.

\section{B. Unsynchronized Diamond Network}

The unsynchronized diamond network is depicted in Fig. 1(b) in Section I-B. The source node transmits a message to the destination node with the help of $K$ parallel relays. The channel inputs at time $t$ transmitted by the source and $k$ th relay are denoted by $x[t]$ and $v_{k}[t]$, respectively. Each channel input passes through an independent $\operatorname{IDC}\left(\mu, \sigma^{2}\right)$. The output of the $k$ th IDC on the first hop is denoted by $\tilde{x}_{k}[\ell]$, while the output of the $k$ th IDC on the second hop is denoted by $\tilde{v}_{k}[\ell]$. The channel outputs at time $\ell$ at the destination and the $k$ th relay are denoted by $y[\ell]$ and $y_{k}[\ell]$, and are related to the IDC outputs as

$$
\begin{aligned}
& y_{k}[\ell] \triangleq \sqrt{g} \tilde{x}_{k}[\ell]+z_{k}[\ell], \\
& y[\ell] \triangleq \sqrt{h} \sum_{k=1}^{K} \tilde{v}_{k}[\ell]+z[\ell],
\end{aligned}
$$

where $\{z[\ell]\}_{\ell},\left\{z_{k}[\ell]\right\}_{k, \ell}$ are independent and identically distributed Gaussian random variables with mean zero and variance one and independent of the corresponding channel inputs. The channel gains $g$ and $h$ are assumed to be real positive numbers, constant as a function of time and known throughout the network. Note that due to the random insertions and deletions, the lengths of the output sequence from different IDCs may not be the same. However, in our achievable scheme, we consider only the first

$$
L \triangleq T \mu
$$

symbols in the output sequence of any IDC, truncating or zero-padding as necessary 1

A $\left(T, M, P_{1}, P_{2}, \varepsilon\right)$ code for the unsynchronized diamond network is a collection of functions

$$
\begin{aligned}
f & :\{1, \ldots, M\} \rightarrow \mathbb{R}^{T}, \\
f_{k} & : \mathbb{R}^{L} \rightarrow \mathbb{R}^{T}, \quad \forall k \in\{1, \ldots, K\}, \\
\phi & : \mathbb{R}^{L} \rightarrow\{1, \ldots, M\},
\end{aligned}
$$

\footnotetext{
${ }^{1}$ To simplify notation, we sometimes assume that quantities such as $\mu T$ are integers and omit $\lfloor\cdot\rfloor$ and $\lceil\cdot\rceil$ operators.
} 
that satisfies the following properties.

- The encoding function $f$ maps the message $w$, assumed to be uniformly distributed over the set $\{1, \ldots, M\}$, to the channel inputs (codeword)

$$
(x[t])_{t=1}^{T} \triangleq f(w)
$$

at the source node.

- The function $f_{k}$ maps the received channel outputs $\left(y_{k}[\ell]\right)_{\ell=1}^{L}$ at the $k$ th relay to its transmitted codeword

$$
\left(v_{k}[t]\right)_{t=1}^{T} \triangleq f_{k}\left(\left(y_{k}[\ell]\right)_{\ell=1}^{L}\right) \text {. }
$$

Note that the function $f_{k}$ is noncausal as described here. See Remark 2 for a discussion.

- The decoding function $\phi$ maps the received channel outputs $(y[\ell])_{\ell=1}^{L}$ at the destination node into a reconstruction

$$
\hat{w} \triangleq \phi\left((y[\ell])_{\ell=1}^{L}\right)
$$

of the message sent by the source node.

- Each codeword satisfies the power constraints

$$
\begin{aligned}
& \frac{1}{T} \sum_{t=1}^{T} x^{2}[t] \leq P_{1}, \\
& \frac{1}{T} \sum_{t=1}^{T} v_{k}^{2}[t] \leq P_{2}, \quad \forall k \in\{1, \ldots, K\} .
\end{aligned}
$$

- The average probability of error satisfies

$$
\mathbb{P}(\hat{w} \neq w) \leq \varepsilon
$$

The total energy spent in transmitting the $\log M$ message bits by the $\left(T, M, P_{1}, P_{2}, \varepsilon\right)$ code is at most $T\left(P_{1}+K P_{2}\right)$. The rate per unit energy achieved by this code is

$$
\frac{\log M}{T\left(P_{1}+K P_{2}\right)},
$$

where here and throughout this paper $\log (\cdot)$ and $\ln (\cdot)$ denote the logarithms to the bases 2 and $e$, respectively.

Definition. A rate of $\hat{R}$ bits per unit energy is achievable if for every $\varepsilon>0$ and every large enough $M$ there exists a $\left(T, M, P_{1}, P_{2}, \varepsilon\right)$ code satisfying

$$
\frac{\log M}{T\left(P_{1}+K P_{2}\right)} \geq \hat{R} .
$$

The capacity per unit energy $\hat{C}$ is the supremum of achievable rates per unit energy.

Remark 1: We emphasize that in the capacity per unit energy setting, the power constraints $P_{1}$ and $P_{2}$ are parameters to be optimized rather than fixed quantities as in the standard capacity setting. This is reflected by the code parameters being denoted by $\left(T, M, P_{1}, P_{2}, \varepsilon\right)$ instead of the usual $(T, M, \varepsilon)$.

Remark 2: To simplify the exposition, we are assuming noncausal encoding functions at the relays. This is not a restriction due to the layered nature of the network. The achievability scheme described in this paper can be adapted to the case of causal encoding functions by delaying the relay transmissions by a predetermined amount of time. This amount of time is chosen to be sufficiently large so that the relay encoding operations become causal. Note that this does not affect the number of bits transmitted per unit energy. A complicating issue is that, since the clocks of the relays are not synchronized with each other, 
the predetermined amount of time may not elapse simultaneously across the relays. As a result, they may start transmitting at different times. However, this mismatch can be handled using the same techniques as in the proof of Theorem 2, The resulting rate per unit energy with causal encoding functions is the same as with noncausal ones.

\section{MAin RESUlTS}

The goal of this paper is to show that the capacity per unit energy of the Gaussian diamond network is decreased by at most a constant factor in the absence of synchronization. To this end, we start with an upper bound on the capacity per unit energy of the synchronized Gaussian diamond network.

Theorem 1. The capacity per unit energy $\hat{C}_{\text {sync }}$ of the synchronized symmetric diamond network with $K$ relays and channel gains $g, h$ is upper bounded by

$$
\hat{C}_{\text {sync }} \leq \frac{2}{\ln 2} \min \{K g, \sqrt{K g h}, K h\} .
$$

The proof of Theorem 1, presented in Section IV] builds on the capacity approximation for the symmetric diamond relay network derived in [13]. The next result states an achievable rate for the unsynchronized Gaussian diamond network.

Theorem 2. The capacity per unit energy $\hat{C}_{\text {unsync }}$ of the unsynchronized symmetric Gaussian diamond network with $K$ relays, channel gains $g, h$, and average clock drift $\mu$ is lower bounded by

$$
\hat{C}_{\text {unsync }} \geq \frac{\mu}{10} \min \{K g, \sqrt{K g h}, K h\} .
$$

The corresponding achievable scheme and the proof of this lower bound are described in Section $\mathrm{V}$ Note that the above result does not depend on the variances of the IDC. In fact, the IDCs need not have the same variance, and we only require that they be finite. Combining Theorems 1 and 2 , we have the following corollary.

Corollary 3. The capacities per unit energy of the synchronized and unsynchronized symmetric Gaussian $K$-relay diamond networks satisfy

$$
\hat{C}_{\text {unsync }} \geq \frac{\mu}{29} \hat{C}_{\text {sync }} .
$$

Corollary 3 states that the loss due to synchronization errors for the Gaussian diamond network is at most a factor $29 / \mu$. As remarked earlier, $\mu \approx 1$ in practice. For those cases, the loss due to synchronization errors is hence at most a factor of approximately 29 . This is perhaps surprising, since tight synchronization between nodes is often thought to be necessary to achieve coherent combining of signals. The result here shows that this tight synchronization is, in fact, not required to achieve essentially the full energy-efficiency gains afforded by beamforming from the relays.

Note that the IDC model used in this paper is physically meaningful only when $\mu \approx 1$, i.e. when the clocks are not wildly out of synchronization. When $\mu \gg 1$, the unsynchronized channel model "creates" additional energy as compared to the synchronized channel, due to the availability of additional independently corrupted channel outputs at the receiver for each channel input. This effect increases the average received energy of the unsynchronized channel compared to the synchronized channel. This explains the fact that Corollary 3 implies that the lower bound on $\hat{C}_{\text {unsync }}$ becomes larger than $\hat{C}_{\text {sync }}$ for $\mu>29$. Thus, it is appropriate to compare $\hat{C}_{\text {unsync }}$ and $\hat{C}_{\text {sync }}$ only when $\mu \approx 1$. For highly unsynchronized clocks, which is uncommon in practice, more sophisticated channel models need to be used. These models need to incorporate aspects of the modulating pulse, among other things, so that when multiple samples are taken, the SNR gets modified appropriately to avoid the artificial creation or destruction of energy.

As a consequence of the above results, we can identify three different operating regimes for both the synchronized and unsynchronized diamond network with respect to the parameters, $g, h$, and $K$. 
- $h<g / K$ : In this regime, the gain $g$ of the source-relay links is much higher than the gain $h$ of the relay-destination links. Under a path-loss model, this would correspond to the relays being located close to the source. By Theorems 1 and 2, the capacity per unit energy is proportional to $K h$.

To understand the improvement provided by the relays, consider a point-to-point channel with a $K$ antenna source and a single-antenna destination with channel gains of magnitude $h$ between them. The $K$ antennas at the source of this point-to-point channel can be used for transmit beamforming, resulting in a capacity per unit energy proportional to $K h$.

We thus see that in this regime the relays of the original diamond relay network can essentially be made to perform as if they were multiple antennas at the source. From [13], we know that the minimum cut in this regime is the multiple-access cut separating the destination from the relays.

- $h \geq K g$ : In this regime, the gain $g$ of the source-relay links are much weaker than the gain $h$ of the relay-destination links. The relays can thus be thought of as being located close to the destination. By Theorems 11 and 2, the capacity per unit energy is proportional to $K g$.

By a similar argument as above, we see that in this regime the relays in the network can essentially be made to perform as if they were multiple antennas at the receiver. From [13], we know that the minimum cut in this regime is the broadcast cut separating the relays from the source.

- $g / K \leq h<K g$ : In this intermediate regime the source-relay gain $g$ is comparable to the relaydestination gain $h$. By Theorem 1 and 2, the capacity per unit energy is proportional to $\sqrt{K g h}$.

To get some insight into this behavior, consider the special case with equal channel gains $g=h$. The capacity per unit energy of the diamond network is then proportional to $\sqrt{K g h}=\sqrt{K} g$. Comparing this to a point-to-point channel with gain $g$, we thus see that the relays in this regime provide a "beamforming" gain that scales as $\sqrt{K}$ instead of $K$. As identified in [13], the minimum cut in this case is neither the multiple-access cut nor the broadcast cut, but rather cuts that include only some of the relays.

Remark 3: We also note that by following the same steps as in the proof of Theorem 2, we can obtain a result similar to Corollary 3 for a model in which the means of the IDCs are not the same. Formally, if the $k$ th IDC in the first hop has mean $\tilde{\mu}_{1, k}$ and the $k$ th IDC in the second hop has mean $\tilde{\mu}_{2, k}$, for $k \in\{1,2, \ldots, K\}$, then we have

$$
\hat{C}_{\text {unsync }} \geq \frac{\tilde{\mu}}{29} \hat{C}_{\text {sync }}
$$

where

$$
\tilde{\mu} \triangleq 2\left(\frac{1}{\min _{1 \leq k \leq K} \tilde{\mu}_{1, k}}+\frac{1}{K} \sum_{k=1}^{K} \frac{1}{\tilde{\mu}_{2, k}}\right)^{-1} .
$$

Elaborating arguments for this case are presented in Section $\mathrm{V}-\mathrm{C}$.

\section{Proof of Theorem 1}

We start by recalling a result from [13, Theorem 2] giving an upper bound on the capacity of the synchronized diamond network.

Theorem 4. For every symmetric diamond network with $K \geq 2$ relays, channel gains $g, h$ and power constraints $P_{1}, P_{2}$, the capacity $C\left(P_{1}, P_{2}\right)$ is upper bounded by

$$
\text { if } \max \left\{P_{1} g, K P_{2} h\right\}<1, P_{1} g \geq K^{2} P_{2} h \text {. }
$$




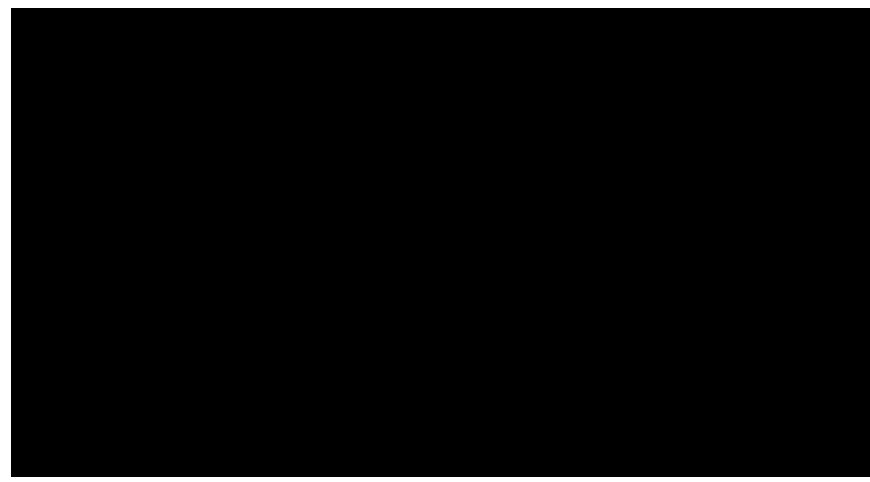

Fig. 2. Different SNR regimes in Theorem 4

Of the five regimes in Theorem 4, cases $(i)$ and $(i i i)$ can be considered as the high-SNR regimes, while $(i i),(i v)$ and $(v)$ can be considered as the low-SNR regimes (see Fig. 2). We start by arguing that we can restrict attention to only the low-SNR regimes.

For power constraints $P_{1}, P_{2}$, denote by $C\left(P_{1}, P_{2}\right)$ the corresponding capacity. The rate per unit energy achievable for fixed powers $\left(P_{1}, P_{2}\right)$ is then

$$
\frac{C\left(P_{1}, P_{2}\right)}{P_{1}+K P_{2}}
$$

and the capacity per unit energy is

$$
\sup _{P_{1}, P_{2} \geq 0} \frac{C\left(P_{1}, P_{2}\right)}{P_{1}+K P_{2}}
$$

We argue that reducing both power constraints by a factor $\lambda \in(0,1)$ cannot decrease this rate per unit energy. Fix such a value of $\lambda$ as well as transmit powers $P_{1}, P_{2}$. Consider communicating during only a fraction $\lambda$ of the total time using the optimal communication scheme for powers $P_{1}$ and $P_{2}$. The rate of communication is $\lambda C\left(P_{1}, P_{2}\right)$, and the average power constraints are $\lambda P_{1}$ and $\lambda P_{2}$. Hence, the achieved rate per unit energy is

$$
\frac{\lambda C\left(P_{1}, P_{2}\right)}{\lambda P_{1}+K \lambda P_{2}}=\frac{C\left(P_{1}, P_{2}\right)}{P_{1}+K P_{2}} .
$$

Since this is only one particular strategy for communicating with average powers $\left(\lambda P_{1}, \lambda P_{2}\right)$, we have

$$
\lambda C\left(P_{1}, P_{2}\right) \leq C\left(\lambda P_{1}, \lambda P_{2}\right),
$$

which implies that

$$
\frac{\lambda C\left(P_{1}, P_{2}\right)}{\lambda P_{1}+K \lambda P_{2}} \leq \frac{C\left(\lambda P_{1}, \lambda P_{2}\right)}{\lambda P_{1}+K \lambda P_{2}}
$$

Combining this, we obtain

$$
\frac{C\left(P_{1}, P_{2}\right)}{P_{1}+K P_{2}} \leq \frac{C\left(\lambda P_{1}, \lambda P_{2}\right)}{\lambda P_{1}+K \lambda P_{2}}
$$

This shows that the capacity per unit energy cannot decrease if $P_{1}$ and $P_{2}$ are replaced by $\lambda P_{1}$ and $\lambda P_{2}$, respectively, for any $0<\lambda<1$. Hence, we can restrict the optimization over transmit powers in (2) to any arbitrarily small neighborhood of the origin. This allows us to ignore the first and third regimes in Theorem 4 (see again Fig. 2).

We now derive an upper bound on the capacity per unit energy in each of the three low-SNR regimes $(i i)$, $(i v)$, and $(v)$. For ease of notation, we do not explicitly state the nonnegativity condition on $P_{1}, P_{2}$ in the following analysis. For the same reason, we also do not state explicitly the condition $\max \left\{P_{1} g, K P_{2} h\right\}<$ 
1, which is common to all the low-SNR regimes, and assume implicitly that $P_{1}$ and $P_{2}$ are small enough to satisfy this condition.

- Assume first that $\left(P_{1}, P_{2}\right)$ are chosen such that we operate in regime $(i v)$, i.e., $P_{2} h<P_{1} g<K^{2} P_{2} h$ and $K \sqrt{P_{1} P_{2} g h}<1$. The achievable rate per unit energy in this regime is upper bounded by (not mentioning the condition $K \sqrt{P_{1} P_{2} g h}<1$ explicitly)

$$
\begin{aligned}
\sup _{\left(P_{1}, P_{2}\right): P_{2} h<P_{1} g<K^{2} P_{2} h} & \frac{\log \left(1+2 K \sqrt{P_{1} P_{2} g h}\right)}{P_{1}+K P_{2}} \\
& \stackrel{(a)}{\leq} \sup _{\left(P_{1}, P_{2}\right): P_{2} h<P_{1} g<K^{2} P_{2} h} \frac{1}{\ln 2} \cdot \frac{2 K \sqrt{P_{1} P_{2} g h}}{P_{1}+K P_{2}} \\
& \stackrel{(b)}{=} \sup _{x: h / g \leq x \leq K^{2} h / g} \frac{2 K \sqrt{g h}}{\ln 2} \cdot \frac{\sqrt{x}}{x+K},
\end{aligned}
$$

where $(a)$ follows from $\ln (1+x) \leq x$ and $(b)$ by making the change of variable $x=P_{1} / P_{2}$. Now, for $x \geq 0$,

$$
\frac{\sqrt{x}}{x+K} \leq \min \left\{\frac{1}{\sqrt{x}}, \frac{\sqrt{x}}{\max \{x, K\}}, \frac{\sqrt{x}}{K}\right\}
$$

so that

$$
\begin{aligned}
\sup _{x: h / g \leq x \leq K^{2} h / g} \frac{\sqrt{x}}{x+K} & \leq \sup _{x: h / g \leq x \leq K^{2} h / g} \min \left\{\frac{1}{\sqrt{x}}, \frac{\sqrt{x}}{\max \{x, K\}}, \frac{\sqrt{x}}{K}\right\} \\
& \leq \min \left\{\frac{\sqrt{g}}{\sqrt{h}}, \frac{1}{\sqrt{K}}, \frac{\sqrt{h}}{\sqrt{g}}\right\} .
\end{aligned}
$$

We thus obtain

$$
\sup _{\left(P_{1}, P_{2}\right): P_{2} h<P_{1} g<K^{2} P_{2} h} \frac{\log \left(1+2 K \sqrt{P_{1} P_{2} g h}\right)}{P_{1}+K P_{2}} \leq \frac{2}{\ln 2} \min \{K g, \sqrt{K g h}, K h\} .
$$

- Assume next that $\left(P_{1}, P_{2}\right)$ are chosen such that we operate in regime $(i i)$, i.e., $P_{1} g \leq P_{2} h$. The achievable rate per unit energy in this regime is upper bounded by

$$
\begin{aligned}
\sup _{\left(P_{1}, P_{2}\right): P_{1} g \leq P_{2} h} & \frac{\frac{1}{2} \log \left(1+K P_{1} g\right)}{P_{1}+K P_{2}} \\
& =\sup _{P_{1}} \sup _{P_{2} \geq P_{1} g / h} \frac{\frac{1}{2} \log \left(1+K P_{1} g\right)}{P_{1}+K P_{2}} \\
& \stackrel{(a)}{=} \frac{1}{2 \ln 2} \cdot \frac{K g}{1+K g / h} \\
& \stackrel{(b)}{\leq} \frac{2}{\ln 2} \min \{K g, \sqrt{K g h}, K h\},
\end{aligned}
$$

where $(a)$ follows by setting $P_{2}=P_{1} g / h$ and letting $P_{1} \rightarrow 0$, and where $(b)$ follows from

$$
\frac{K g}{1+K g / h} \leq \sqrt{K g h} / 2
$$

which can be proved using the arithmetic-mean-geometric-mean inequality. 
- Assume finally that $\left(P_{1}, P_{2}\right)$ are chosen such that we operate in regime $(v)$, i.e., $P_{1} g \geq K^{2} P_{2} h$. The achievable rate per unit energy in this regime is upper bounded by

$$
\begin{aligned}
\sup _{\left(P_{1}, P_{2}\right): P_{1} g \geq K^{2} P_{2} h} & \frac{\frac{1}{2} \log \left(1+K^{2} P_{2} h\right)}{P_{1}+K P_{2}} \\
& =\sup _{P_{2}} \sup _{P_{1} \geq K^{2} P_{2} h / g} \frac{\frac{1}{2} \log \left(1+K^{2} P_{2} h\right)}{P_{1}+K P_{2}} \\
& \stackrel{(a)}{=} \frac{1}{2 \ln 2} \cdot \frac{K h}{1+K h / g} \\
& \stackrel{(b)}{\leq} \frac{2}{\ln 2} \min \{K g, \sqrt{K g h}, K h\},
\end{aligned}
$$

where $(a)$ follows by setting $P_{1}=K^{2} P_{2} h / g$ and letting $P_{2} \rightarrow 0$, and where $(b)$ follows again by (3). The achievable rate per unit energy is thus upper bounded by

$$
\frac{2}{\ln 2} \min \{K g, \sqrt{K g h}, K h\},
$$

in all three low-SNR regimes $(i i),(i v)$, and $(v)$, concluding the proof of Theorem 1

\section{PROOF OF THEOREM 2}

The basic construction of the achievable scheme is as follows. The transmitters employ a codebook in which each codeword consists of a series of long and low-power pulses. This codebook can be described as the concatenation of an outer random code with an inner repetition code. The length of the pulses ensures robustness of the codewords with respect to insertions/deletions and the low power ensures energy efficiency of the communication. In order to achieve low error probability and high energy efficiency, every receiving node needs to be reasonably certain about the location of the different pulses in the transmitted signal and there should be coherent combining of the relay transmissions at the destination. We meet these two requirements by choosing the code parameters appropriately.

Encoding at the source, processing at the relays, and decoding at the destination are illustrated in Fig. 3 . We provide a detailed description of the scheme in Section $\mathrm{V}-\mathrm{A}$. The analysis of this scheme is contained in Section $\mathrm{V}-\mathrm{B}$. We point out that we make conservative choices for the block length to keep the analysis simple. The block length can be made significantly smaller than presented in the following analysis at the cost of a more complicated analysis.

\section{A. Construction}

Encoding at the Source: The source first constructs an outer code by randomly and independently generating $(u[n])_{n=1}^{N}$ for each message $w \in\{1,2, \ldots, M\}$ according to the uniform distribution over the set $\left\{-\sqrt{P_{1}}, \sqrt{P_{1}}\right\}^{N}$. For simplicity of notation, we do not explicitly indicate the dependence of the sequence $(u[n])_{n=1}^{N}$ on the message $w$. The energy of each of these sequences is $N P_{1}$. To form the transmission sequences, the source then employs an inner code.

This inner code is a simple repetition code, attenuated so that the total energy remains unchanged. Specifically, the channel inputs $(x[t])_{t=1}^{T}$ with

$$
T \triangleq N^{5}
$$

are formed by

- repeating $N^{\prime}$ times each symbol in $(u[n])_{n=1}^{N}$, where

$$
N^{\prime} \triangleq N^{4},
$$




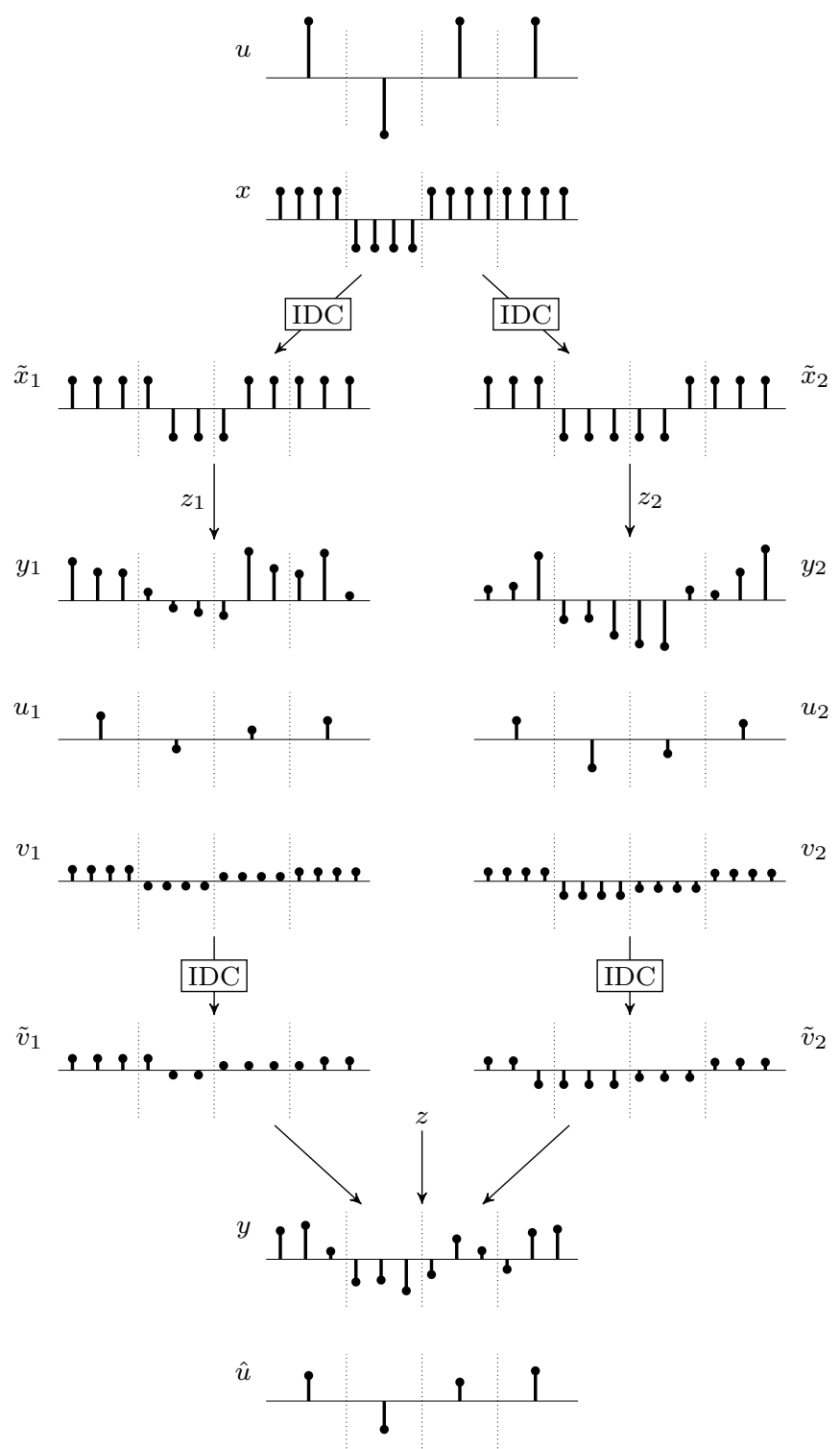

Fig. 3. An illustration of the achievable scheme for a two-relay diamond network with IDC parameter $\mu=3 / 4$. The notation is the same as in Fig. 1(b) in Section I-B In the figure, we use an outer code of block length $N=4$ (see signals $u, u_{k}$, and $\hat{u}$ ) together with an inner repetition code of block length $N^{\prime}=4$ (see signals $x$ and $v_{k}$ ). The block length of the combined code is $T=N N^{\prime}=16$. Each relay forms an estimate of the symbols in the outer code by averaging every $\mu N^{\prime}=3$ consecutive channel outputs (see the transformation from $y_{k}$ to $u_{k}$ ). This estimate is then re-encoded using the same inner repetition code (see the transformation from $u_{k}$ to $v_{k}$ ). The destination uses the same averaging to compute an estimate $\hat{u}$ of the symbols in the outer code. The decoding of the outer code itself is done using the maximum-likelihood sequence detector. Note that only the destination, but not the relays, decode the outer code.

and

- attenuating by a factor $1 / \sqrt{N^{\prime}}$.

As a result, the channel inputs are piecewise constant sequences containing $N$ pieces, each piece having length equal to $N^{\prime}$, and height either $+\sqrt{P_{1} / N^{\prime}}$ or $-\sqrt{P_{1} / N^{\prime}}$.

Formally, the $t$ th channel input $x[t]$ is given by

$$
x[t] \triangleq \frac{1}{\sqrt{N^{\prime}}} u\left[\left\lfloor\frac{t-1}{N^{\prime}}\right\rfloor+1\right]
$$

for all $t \in\{1,2, \ldots, T\}$. The length of each channel input is $T=N N^{\prime}=N^{5}$, and the total energy used at the source is

$$
N N^{\prime} \cdot \frac{1}{N^{\prime}} \cdot P_{1}=N P_{1}
$$


Processing at the Relays: Relay $k$ divides its received signal $\left(y_{k}[\ell]\right)_{\ell=1}^{L}$ with $L=\mu T$ into $N$ consecutive blocks of length $\mu N^{\prime}$. It forms a normalized sum of the received signal in each block resulting in the sequence $\left(u_{k}[n]\right)_{n=1}^{N}$ of length $N$. Formally, the $n$th symbol $u_{k}[n]$ is given by

$$
u_{k}[n] \triangleq \frac{1}{\sqrt{\mu N^{\prime}}} \sum_{\ell=(n-1) \mu N^{\prime}+1}^{n \mu N^{\prime}} y_{k}[\ell]
$$

for all $n \in\{1,2, \ldots, N\}$.

Relay $k$ then forms its own transmit sequence $\left(v_{k}[t]\right)_{t=1}^{T}$ using the attenuated repetition code, except that instead of attenuating by a factor $1 / \sqrt{N^{\prime}}$, it uses the attenuation factor $\alpha / \sqrt{N^{\prime}}$, where

$$
\alpha \triangleq \sqrt{\frac{P_{2}}{1+\mu g P_{1}}} .
$$

Formally,

$$
v_{k}[t] \triangleq \frac{\alpha}{\sqrt{N^{\prime}}} u_{k}\left[\left\lfloor\frac{t-1}{N^{\prime}}\right\rfloor+1\right]
$$

for all $t \in\{1,2, \ldots, T\}$.

The amplification factor is chosen to limit the energy spent at the relay. Indeed, the total energy spent by each relay is no more than

$$
N N^{\prime} \cdot \frac{\alpha^{2}}{N^{\prime}} \cdot\left(\mu g P_{1}+1\right)=N P_{2},
$$

where the +1 term accounts for the forwarded receiver noise.

Decoding at the Destination: The destination performs a similar operation as the relays. It divides its received signal $(y[\ell])_{\ell=1}^{L}$ into $N$ consecutive blocks of length $\mu N^{\prime}$. It forms the normalized sum of the received signal in each block resulting in the sequence $(\hat{u}[n])_{n=1}^{N}$ of length $N$. Formally,

$$
\hat{u}[n] \triangleq \frac{1}{\sqrt{\mu N^{\prime}}} \sum_{\ell=(n-1) \mu N^{\prime}+1}^{n \mu N^{\prime}} y[\ell]
$$

for all $n \in\{1,2, \ldots, N\}$.

The destination considers the end-to-end channel from $(u[n])_{n=1}^{N}$ to $(\hat{u}[n])_{n=1}^{N}$ established as a result of the operations described above and attempts to decode the transmitted message using maximum-likelihood decoding.

\section{B. Analysis}

The analysis of the probability of error consists of the following key steps.

- The randomness of the IDCs causes the constant pieces in the first-hop-IDC outputs $\tilde{x}_{k}$ to not align perfectly with the blocks that the relays divide their received signal into, e.g., the constant pieces in $\tilde{x}_{k}$ in Fig. 3 do not align with the dotted vertical lines. This manifests as inter-symbol interference (ISI) in the signal $u_{k}$. The first step is to show that this ISI is negligible as $N \rightarrow \infty$.

- For high energy efficiency, it is crucial to achieve a beamforming gain at the destination. To this end, we argue that, as $N \rightarrow \infty$, the $n$th piece in every second-hop IDC output $\tilde{v}_{k}$ approximately align at the destination.

- To operate within a constant factor of the optimal energy efficiency, the values of $P_{1}$ and $P_{2}$ need to be chosen appropriately depending on the system parameters $g, h, K$.

- Finally, the restriction to the binary alphabet $\left\{-\sqrt{P_{1}}, \sqrt{P_{1}}\right\}$ (which simplifies the analysis of ISI) results in a rate loss, which needs to be bounded. 
Fix a target error probability $\varepsilon>0$. Denote by $\mathcal{E}$ the decoding error event, and let $\mathcal{E}_{\text {IDC }}$ denote the event that any piece in the output of any IDC behaves far from expectation (defined formally below). The probability of error $\mathbb{P}(\mathcal{E})$ can be upper bounded as

$$
\begin{aligned}
\mathbb{P}(\mathcal{E}) & =\mathbb{P}\left(\mathcal{E} \mid \mathcal{E}_{\mathrm{IDC}}\right) \mathbb{P}\left(\mathcal{E}_{\mathrm{IDC}}\right)+\mathbb{P}\left(\mathcal{E} \mid \mathcal{E}_{\mathrm{IDC}}^{c}\right) \mathbb{P}\left(\mathcal{E}_{\mathrm{IDC}}^{c}\right) \\
& \leq \mathbb{P}\left(\mathcal{E}_{\mathrm{IDC}}\right)+\mathbb{P}\left(\mathcal{E} \mid \mathcal{E}_{\mathrm{IDC}}^{c}\right)
\end{aligned}
$$

We next argue that, for large enough $N$, each of these two terms can be made less than $\varepsilon / 2$.

\section{Behavior of the IDCs}

We start with the analysis of the term $\mathbb{P}\left(\mathcal{E}_{\mathrm{IDC}}\right)$ in $(8)$. To this end, we establish that the behavior of every IDC in the network concentrates around its expectation.

Let $\left(s_{k}[t]\right)_{t=1}^{T}$ denote the state sequence of the $k$ th IDC. The input sequence to any IDC is a piecewise constant sequence containing $N$ pieces. Each piece has length $N^{\prime}$ and the $n$th piece starts at position $(n-1) N^{\prime}+1$ and ends at position $n N^{\prime}$. The output of the IDC is also a piecewise constant sequence, but the pieces now have random lengths with expected value $N^{\prime} \mu$.

Consider the $n$th piece in the output of the $k$ th IDC. Let $\mathcal{E}_{k, n}$ denote the event that this piece starts outside the interval

$$
\left((n-1) \mu N^{\prime}+1-\nu:(n-1) \mu N^{\prime}+1+\nu\right)
$$

or has length outside the interval

$$
\left(\mu N^{\prime}-\beta: \mu N^{\prime}+\beta\right)
$$

where

$$
\nu \triangleq N^{7 / 2}
$$

and

$$
\beta \triangleq N^{3}
$$

Here and in the remainder of this paper we use the notation $[a: b]$ to denote all integers in the closed interval $[a, b]$, i.e. $[a: b]=[a, b] \cap \mathbb{Z}$, and similar for $(a: b)$. Denote by

$$
\mathcal{E}_{\mathrm{IDC}} \triangleq \cup_{k=1}^{2 K} \cup_{n=1}^{N} \mathcal{E}_{k, n}
$$

the event that any piece in the output of any IDC behaves far from expectation

The probability that the $n$th piece in the output of the $k$ th IDC starts outside the interval

$$
\left((n-1) \mu N^{\prime}+1-\nu:(n-1) \mu N^{\prime}+1+\nu\right)
$$

is equal to

$$
\begin{aligned}
\mathbb{P}\left(\left|\sum_{t=1}^{(n-1) N^{\prime}} s_{k}[t]-(n-1) \mu N^{\prime}\right| \geq \nu\right) & \stackrel{(a)}{\leq} \frac{(n-1) N^{\prime} \sigma^{2}}{\nu^{2}} \\
& \leq \frac{N N^{\prime} \sigma^{2}}{\nu^{2}} \\
& \stackrel{(b)}{=} \frac{N^{5} \sigma^{2}}{\left(N^{7 / 2}\right)^{2}} \\
& =\frac{\sigma^{2}}{N^{2}}
\end{aligned}
$$

where $(a)$ follows from Chebyshev's inequality and the definition of $\mu$ and $\sigma^{2}$ as the mean and variance of $s_{k}[t]$, and where $(b)$ follows from the definition of $N^{\prime}$ in (4). 
The probability that the $n$th piece in the output of the $k$ th IDC has length outside

$$
\left(\mu N^{\prime}-\beta: \mu N^{\prime}+\beta\right)
$$

is equal to

$$
\begin{aligned}
\mathbb{P}\left(\left|\sum_{t=(n-1) N^{\prime}+1}^{n N^{\prime}} s_{i}[t]-\mu N^{\prime}\right| \geq \beta\right) & \stackrel{(a)}{\leq} \frac{N^{\prime} \sigma^{2}}{\beta^{2}} \\
& =\frac{N^{4} \sigma^{2}}{\left(N^{3}\right)^{2}} \\
& =\frac{\sigma^{2}}{N^{2}},
\end{aligned}
$$

where $(a)$ follows again from Chebyshev's inequality.

Hence, by the union bound,

$$
\mathbb{P}\left(\mathcal{E}_{k, n}\right) \leq \frac{2 \sigma^{2}}{N^{2}} .
$$

By the union bound again, we thus have

$$
\begin{aligned}
\mathbb{P}\left(\mathcal{E}_{\mathrm{IDC}}\right) & =\mathbb{P}\left(\cup_{k=1}^{2 K} \cup_{n=1}^{N} \mathcal{E}_{k, n}\right) \\
& \leq \sum_{k=1}^{2 K} \sum_{n=1}^{N} \mathbb{P}\left(\mathcal{E}_{k, n}\right) \\
& \leq \frac{4 K \sigma^{2}}{N} \\
& \leq \varepsilon / 2
\end{aligned}
$$

for $N$ large enough.

Analysis of the Received SNR on the End-to-End Channel

We continue with the analysis of the term $\mathbb{P}\left(\mathcal{E} \mid \mathcal{E}_{\mathrm{IDC}}^{c}\right)$ in $(8)$. We will argue that this term is also upper bounded by $\varepsilon / 2$ for rate per unit energy less than or equal to

$$
\frac{\mu}{10} \min \{K g, \sqrt{K g h}, K h\}
$$

and $N$ large enough. For this purpose, we provide a worst-case lower bound on the received SNR of every symbol of the outer code. This allows us to lower bound the achievable rate, which in turn leads to the aforementioned lower bound on the capacity per unit energy.

First Hop: For the analysis of the first hop, number the IDCs from the source to the relays as 1 through $K$. Consider the operation of relay $k$ on the received signal. Without loss of generality, assume that the $n$th symbol in the outer code at the source is

$$
u[n]=+\sqrt{P_{1}} .
$$

The relay sums up its received signal in the block

$$
\left[(n-1) \mu N^{\prime}+1: n \mu N^{\prime}\right]
$$


to form $u_{k}[n]$, which is treated as the noise-corrupted signal corresponding to the transmitted symbol $u[n]]^{2}$

$$
\begin{aligned}
u_{k}[n] & =\frac{1}{\sqrt{\mu N^{\prime}}} \sum_{\ell=(n-1) \mu N^{\prime}+1}^{n \mu N^{\prime}} y_{k}[\ell] \\
& =\frac{1}{\sqrt{\mu N^{\prime}}} \sum_{\ell=(n-1) \mu N^{\prime}+1}^{n \mu N^{\prime}}\left(\sqrt{g} \tilde{x}_{k}[\ell]+z_{k}[\ell]\right)
\end{aligned}
$$

Using $\hat{z}_{k}[n]$ to denote the quantity

$$
\hat{z}_{k}[n] \triangleq \frac{1}{\sqrt{\mu N^{\prime}}} \sum_{\ell=(n-1) \mu N^{\prime}+1}^{n \mu N^{\prime}} z_{k}[\ell] \sim \mathcal{N}(0,1),
$$

we can rewrite the expression for $u_{k}[n]$ as

$$
u_{k}[n]=\left(\sqrt{\frac{g}{\mu N^{\prime}}} \sum_{\ell=(n-1) \mu N^{\prime}+1}^{n \mu N^{\prime}} \tilde{x}_{k}[\ell]\right)+\hat{z}_{k}[n] .
$$

Similarly,

$$
\begin{aligned}
& u_{k}[n-1]=\left(\sqrt{\frac{g}{\mu N^{\prime}}} \sum_{\ell=(n-2) \mu N^{\prime}+1}^{(n-1) \mu N^{\prime}} \tilde{x}_{k}[\ell]\right)+\hat{z}_{k}[n-1], \\
& u_{k}[n+1]=\left(\sqrt{\frac{g}{\mu N^{\prime}}} \sum_{\ell=n \mu N^{\prime}+1}^{(n+1) \mu N^{\prime}} \tilde{x}_{k}[\ell]\right)+\hat{z}_{k}[n+1] .
\end{aligned}
$$

The input to any IDC is a piecewise constant signal where the pieces

$$
\left[(n-1) N^{\prime}+1: n N^{\prime}\right]
$$

align precisely with the blocks

$$
\left[(n-1) N^{\prime}+1: n N^{\prime}\right]
$$

However, due to the randomness of the IDC, the $n$th piece in the output of the IDC may not align with the $n$th output block

$$
\left[(n-1) \mu N^{\prime}+1: n \mu N^{\prime}\right]
$$

This block can contain signal resulting from neighboring pieces in the input sequence, which correspond to $u[n-1]$ and $u[n+1]$. Since relay $k$ forms $u_{k}[n]$ according to (12), this leakage from neighboring symbols manifests itself as inter-symbol interference. However, conditioned on $\mathcal{E}_{\text {IDC }}^{c}$, we expect that the block

$$
\left[(n-1) \mu N^{\prime}+1: n \mu N^{\prime}\right]
$$

contains a significant portion of the $n$th piece so that the inter-symbol interference is low. This is indeed the case as formalized below.

\footnotetext{
${ }^{2}$ To maintain a clear and consistent terminology, we use "piece" to denote an interval over which the signal is constant, and "block" to denote an interval of the form $\left[(n-1) N^{\prime}+1: n N^{\prime}\right]$ or $\left[(n-1) \mu N^{\prime}+1: n \mu N^{\prime}\right]$, depending on whether it is the input or output of an IDC, respectively. To differentiate between the signal component from the noise component in the received signal, we use "signal" to denote only the component due to source transmission, and use "received signal" to denote signal plus any noise component.
} 
For the worst inter-symbol interference, we assume that the neighboring symbols $u[n-1]$ and $u[n+1]$ are both equal to $-u[n]=-\sqrt{P_{1}}$. Conditioned on $\mathcal{E}_{\text {IDC }}^{c}$, we know that the $n$th piece in the output of the IDC starts in the interval

$$
\left((n-1) \mu N^{\prime}+1-\nu:(n-1) \mu N^{\prime}+1+\nu\right)
$$

and has length in the interval

$$
\left(\mu N^{\prime}-\beta: \mu N^{\prime}+\beta\right)
$$

Hence the block

$$
\left[(n-1) \mu N^{\prime}+1: n \mu N^{\prime}\right]
$$

in the output of the IDC contains a constant piece of length at least $\mu N^{\prime}-\nu-\beta$ and height $u[n] / \sqrt{N^{\prime}}$. The remaining at most $\nu+\beta$ symbols in the block

$$
\left[(n-1) \mu N^{\prime}+1: n \mu N^{\prime}\right]
$$

are each equal to $-u[n] / \sqrt{N^{\prime}}$. Hence, we have the following lower bound on the signal in $u_{k}[n]$ (the term inside parentheses in (12)):

$$
\begin{aligned}
\sqrt{\frac{g}{\mu N^{\prime}}} \sum_{\ell=(n-1) \mu N^{\prime}+1}^{n \mu N^{\prime}} \tilde{x}_{k}[\ell] & \geq \sqrt{\frac{g}{\mu N^{\prime}}}\left(\mu N^{\prime}-\nu-\beta\right) \frac{u[n]}{\sqrt{N^{\prime}}}+\sqrt{\frac{g}{\mu N^{\prime}}}(\nu+\beta) \frac{-u[n]}{\sqrt{N^{\prime}}} \\
& =\sqrt{\mu g}\left(1-\frac{2 \nu+2 \beta}{\mu N^{\prime}}\right) u[n] \\
& =\sqrt{\mu g}\left(1-2 \delta_{N}\right) \sqrt{P_{1}},
\end{aligned}
$$

where

$$
\delta_{N} \triangleq \frac{\nu+\beta}{\mu N^{\prime}}=\frac{N^{7 / 2}+N^{3}}{\mu N^{4}} \rightarrow 0
$$

as $N \rightarrow \infty$ using the definitions of $\nu$ and $\beta$ in (9) and (10).

We also have the simple upper bound

$$
\sqrt{\frac{g}{\mu N^{\prime}}} \sum_{\ell=(n-1) \mu N^{\prime}+1}^{n \mu N^{\prime}} \tilde{x}_{k}[\ell] \leq \sqrt{\mu g} \cdot \sqrt{P_{1}} .
$$

obtained by assuming that there is no inter-symbol interference.

Similarly, since $u[n-1]=u[n+1]=-u[n]=-\sqrt{P_{1}}$, we can derive the analogous bounds

$$
\begin{gathered}
\sqrt{\frac{g}{\mu N^{\prime}}} \sum_{\ell=(n-2) \mu N^{\prime}+1}^{(n-1) \mu N^{\prime}} \tilde{x}_{k}[\ell] \geq-\sqrt{\mu g} \cdot \sqrt{P_{1}}, \\
\sqrt{\frac{g}{\mu N^{\prime}}} \sum_{\ell=n \mu N^{\prime}+1}^{(n+1) \mu N^{\prime}} \tilde{x}_{k}[\ell] \geq-\sqrt{\mu g} \cdot \sqrt{P_{1}}
\end{gathered}
$$

for the signal in $u_{k}[n-1]$ and $u_{k}[n+1]$ (the terms inside parentheses in (13) and (14), respectively). 
Second Hop: For the analysis of the second hop, number the IDCs from the relays to the destination as 1 through $K$. By the definition of $\hat{u}[n]$,

$$
\begin{aligned}
\hat{u}[n] & =\frac{1}{\sqrt{\mu N^{\prime}}} \sum_{\ell=(n-1) \mu N^{\prime}+1}^{n \mu N^{\prime}} y[\ell] \\
& =\frac{1}{\sqrt{\mu N^{\prime}}} \sum_{\ell=(n-1) \mu N^{\prime}+1}^{n \mu N^{\prime}}\left(\sqrt{h} \sum_{k=1}^{K} \tilde{v}_{k}[\ell]+z[\ell]\right) \\
& =\sqrt{\frac{h}{\mu N^{\prime}}} \sum_{k=1}^{K} \sum_{\ell=(n-1) \mu N^{\prime}+1}^{n \mu N^{\prime}} \tilde{v}_{k}[\ell]+\hat{z}[n],
\end{aligned}
$$

where similar to before

$$
\hat{z}[n] \triangleq \frac{1}{\sqrt{\mu N^{\prime}}} \sum_{\ell=(n-1) \mu N^{\prime}+1}^{n \mu N^{\prime}} z[\ell] \sim \mathcal{N}(0,1) .
$$

Consider the $k$ th IDC. The input to this IDC is a piecewise constant sequence, where the $n$th piece, with value $\frac{\alpha}{\sqrt{N^{\prime}}} u_{k}[n]$, aligns precisely with the block

$$
\left[(n-1) N^{\prime}+1: n N^{\prime}\right]
$$

However, as before, the $n$th piece in the output of the IDC may not align with the block

$$
\left[(n-1) \mu N^{\prime}+1: n \mu N^{\prime}\right]
$$

due to the randomness of the IDC. Some of the output symbols in this block could be equal to $\frac{\alpha}{\sqrt{N^{\prime}}} u_{k}[n-1]$ or $\frac{\alpha}{\sqrt{N^{\prime}}} u_{k}[n+1]$ causing inter-symbol interference. However, since we condition on $\mathcal{E}_{\text {IDC }}^{c}$, we know that this block contains a piece of length at least $\mu N^{\prime}-\nu-\beta$ and height $\frac{\alpha}{\sqrt{N^{\prime}}} \cdot u_{k}[n]$, and the remaining at most $\nu+\beta$ symbols are equal to either $\frac{\alpha}{\sqrt{N^{\prime}}} \cdot u_{k}[n-1]$ or $\frac{\alpha}{\sqrt{N^{\prime}}} \cdot u_{k}[n+1]$.

For concreteness, assume that the number of symbols in this block that take values $\frac{\alpha}{\sqrt{N^{\prime}}} \cdot u_{k}[n-1]$, $\frac{\alpha}{\sqrt{N^{\prime}}} \cdot u_{k}[n]$ and $\frac{\alpha}{\sqrt{N^{\prime}}} \cdot u_{k}[n+1]$ are $L_{k, n-1}, L_{k, n}$, and $L_{k, n+1}$, respectively. From the above discussion, note that

$$
L_{k, n} \geq \mu N^{\prime}-\nu-\beta
$$

and

$$
L_{k, n-1}+L_{k, n+1} \leq \nu+\beta
$$

Continuing (20), we thus have

$$
\begin{aligned}
& \hat{u}[n]=\sqrt{\frac{h}{\mu N^{\prime}}} \sum_{k=1}^{K} \sum_{\ell=(n-1) \mu N^{\prime}+1}^{n \mu N^{\prime}} \tilde{v}_{k}[\ell]+\hat{z}[n] \\
& =\sqrt{\frac{h}{\mu N^{\prime}}} \sum_{k=1}^{K}\left(L_{k, n} \frac{\alpha}{\sqrt{N^{\prime}}} u_{k}[n]+L_{k, n-1} \frac{\alpha}{\sqrt{N^{\prime}}} u_{k}[n-1]+L_{k, n+1} \frac{\alpha}{\sqrt{N^{\prime}}} u_{k}[n+1]\right)+\hat{z}[n] \\
& \geq \sqrt{\frac{h}{\mu N^{\prime}}} \sum_{k=1}^{K}\left(L_{k, n} \frac{\alpha}{\sqrt{N^{\prime}}}\left(\left(1-2 \delta_{N}\right) \sqrt{\mu g P_{1}}+\hat{z}_{k}[n]\right)\right. \\
& \left.+L_{k, n-1} \frac{\alpha}{\sqrt{N^{\prime}}}\left(-\sqrt{\mu g P_{1}}+\hat{z}_{k}[n-1]\right)+L_{k, n+1} \frac{\alpha}{\sqrt{N^{\prime}}}\left(-\sqrt{\mu g P_{1}}+\hat{z}_{k}[n+1]\right)\right)+\hat{z}[n],
\end{aligned}
$$

where we have used (12), (13), (14), (15), (18), and (19). 
Simplifying and using (16), (21), and (22) yields

$$
\begin{aligned}
\hat{u}[n] \geq & K \alpha \mu \sqrt{g h}\left(1-4 \delta_{N}\right) \sqrt{P_{1}} \\
& +\sqrt{\frac{h}{\mu N^{\prime}}} \cdot \frac{\alpha}{\sqrt{N^{\prime}}} \sum_{k=1}^{K}\left(L_{k, n-1} \hat{z}_{k}[n-1]+L_{k, n} \hat{z}_{k}[n]+L_{k, n+1} \hat{z}_{k}[n+1]\right)+\hat{z}[n] .
\end{aligned}
$$

The second term in the last expression constitutes the end-to-end noise. It is clear that it is normal with zero mean. The term inside parentheses is independent of $\hat{z}[n] \sim \mathcal{N}(0,1)$. We can upper bound the variance of the term in parentheses by assuming $L_{k, n}=\mu N^{\prime}$ and $L_{k, n-1}=L_{k, n+1}=0$ for all $k$. The total noise power is then upper bounded by

$$
\begin{aligned}
\mathbb{E}\left(\left(\sqrt{\frac{h}{\mu N^{\prime}}} \cdot \frac{\alpha}{\sqrt{N^{\prime}}} \sum_{k=1}^{K} \mu N^{\prime} \hat{z}_{k}[n]+\hat{z}[n]\right)^{2}\right) & =\frac{h}{\mu N^{\prime}} \cdot \frac{\alpha^{2}}{N^{\prime}} K\left(\mu N^{\prime}\right)^{2}+1 \\
& =K \alpha^{2} \mu h+1
\end{aligned}
$$

Note that the noise in the received signal $(\hat{u}[n])_{n=1}^{N}$ is not i.i.d. across $n$ due to the leakage of the firsthop noise from neighboring pieces, i.e., $\hat{z}_{k}[n]$ appears in the noise expressions for blocks $n-1, n$, and $n+1$. However, we can ignore this fact and assume that the noise is independent across blocks, because by interleaving codewords of the outer code such that successive symbols of a codeword are sufficiently apart, they can be made to experience independent noise. This allows us to treat the channel as an AWGN channel. A scheme that makes use of the dependence of noise across blocks can potentially achieve higher rates, but for simplicity of analysis we restrict attention to schemes that interleave codewords and treat noise as independent across blocks.

The end-to-end SNR of the effective AWGN channel is at least

$$
\frac{\left(K \alpha \mu \sqrt{g h P_{1}}\left(1-4 \delta_{N}\right)\right)^{2}}{K \alpha^{2} \mu h+1} \geq \frac{K^{2} \mu^{2} g h P_{1} P_{2}}{1+\mu g P_{1}+K \mu h P_{2}}\left(1-8 \delta_{N}\right),
$$

where we have used the definition of $\alpha=\sqrt{P_{2} /\left(1+\mu g P_{1}\right)}$ in (6). Since $\delta_{N} \rightarrow 0$ as $N \rightarrow \infty$ by (16), we have that the SNR is lower bounded by $\mathrm{SNR}_{l b}$, where $\mathrm{SNR}_{l b}$ is given by

$$
\mathrm{SNR}_{l b} \triangleq \frac{K^{2} \mu^{2} P_{1} P_{2} g h}{1+\mu g P_{1}+K \mu h P_{2}} .
$$

Let $C_{\text {binary }}(\mathrm{SNR})$ denote the largest achievable rate with binary antipodal constellation of the AWGN channel as a function of SNR, and $C(\mathrm{SNR})=\frac{1}{2} \log (1+\mathrm{SNR})$ denote the capacity of the AWGN channel. Since the achievability scheme employs a binary antipodal constellation, the largest rate that can be achieved is given by $C_{\text {binary }}(\mathrm{SNR})$ which, by (23), is lower-bounded by

$$
C_{\text {binary }}\left(\mathrm{SNR}_{l b}\right) \text {, }
$$

which we will show later can be further lower bounded by

$$
\gamma C\left(\mathrm{SNR}_{l b}\right)=\frac{\gamma}{2} \log \left(1+\mathrm{SNR}_{l b}\right),
$$

for all $\mathrm{SNR}_{l b}$ of interest, where $\gamma$ is a constant.

Using this, the achievable scheme we have considered can transmit at a rate arbitrarily close to

$$
\frac{\gamma}{2} \log \left(1+\mathrm{SNR}_{l b}\right)
$$

bits per channel use with probability of error

$$
\mathbb{P}\left(\mathcal{E} \mid \mathcal{E}_{\text {IDC }}^{c}\right)<\varepsilon / 2
$$

for sufficiently large $N$. 


\section{Rate per Unit Energy}

Substituting (11) and (25) into (8) shows that the overall probability of error is at most $\mathbb{P}(\mathcal{E})<\varepsilon$ for $N$ large enough. Since the total energy used during transmission is no more than $N P_{1}+K N P_{2}$ by (5) and (7), the achieved bits per unit energy is

$$
\begin{aligned}
& \geq \frac{N C_{\text {binary }}(\mathrm{SNR})}{N\left(P_{1}+K P_{2}\right)} \\
& \geq \frac{C_{\text {binary }}\left(\mathrm{SNR}_{l b}\right)}{P_{1}+K P_{2}} \\
& \geq \frac{\gamma C\left(\mathrm{SNR}_{l b}\right)}{P_{1}+K P_{2}} \\
& =\frac{\frac{\gamma}{2} \log \left(1+\frac{K^{2} \mu^{2} g h P_{1} P_{2}}{1+\mu g P_{1}+K \mu h P_{2}}\right)}{P_{1}+K P_{2}} .
\end{aligned}
$$

We now choose $P_{1}$ and $P_{2}$ depending on the regime of $(g, h, K)$. We consider the cases $h<g / K$, $g / K \leq h<K g$, and $h \geq K g$ separately.

- If $h<g / K$, we choose

$$
\begin{aligned}
& P_{1}=\frac{1}{\mu g}, \\
& P_{2}=\frac{1}{\mu K^{2} h} .
\end{aligned}
$$

Then the achievable bits per energy are

$$
\frac{\frac{\gamma}{2} \log \left(1+\frac{1}{1+1+1 / K}\right)}{1 /(\mu g)+1 /(\mu K h)} \geq \frac{\gamma \mu \log (4 / 3)}{4} K h,
$$

where we have used $h<g / K$.

- If $g / K \leq h<K g$, we choose

$$
\begin{aligned}
& P_{1}=\frac{1}{\mu \sqrt{K g h}}, \\
& P_{2}=\frac{1}{\mu \sqrt{K^{3} g h}} .
\end{aligned}
$$

Then the achievable bits per energy are

$$
\begin{aligned}
\frac{\frac{\gamma}{2} \log \left(1+\frac{1}{1+\sqrt{g /(K h)}+\sqrt{h /(K g)}}\right)}{1 /(\mu \sqrt{K g h})+1 /(\mu \sqrt{K g h})} & =\frac{\gamma \mu \sqrt{K g h}}{4} \log \left(1+\frac{1}{1+\sqrt{g /(K h)}+\sqrt{h /(K g)}}\right) \\
& \stackrel{(a)}{\geq} \frac{\gamma \mu \log (4 / 3)}{4} \sqrt{K g h},
\end{aligned}
$$

where we have used $1 / \sqrt{K} \leq \sqrt{h / g}<\sqrt{K}$.

- If $h \geq K g$, we choose

$$
\begin{aligned}
& P_{1}=\frac{1}{\mu K g}, \\
& P_{2}=\frac{1}{\mu K h} .
\end{aligned}
$$


Then the achievable bits per energy are

$$
\frac{\frac{\gamma}{2} \log \left(1+\frac{1}{1+1 / K+1}\right)}{1 /(\mu K g)+1 /(\mu h)} \geq \frac{\gamma \mu \log (4 / 3)}{4} K g,
$$

where we have used $h \geq K g$.

Hence from (26), (27) and (28), we conclude that the rate per unit energy achieved by the proposed scheme is at least

$$
\begin{aligned}
& \frac{\gamma \mu \log (4 / 3)}{4} \cdot \begin{cases}K h, & \text { if } h<g / K, \\
\sqrt{K g h}, & \text { if } g / K \leq h<K g, \\
K g, & \text { if } h \geq K g .\end{cases} \\
& \geq \frac{\gamma \mu \log (4 / 3)}{4} \cdot \min \{K g, \sqrt{K g h}, K h\} .
\end{aligned}
$$

It remains only to find the constant $\gamma$ in (24). We would like to choose $\gamma$ as large as possible such that $C_{\text {binary }}\left(\mathrm{SNR}_{l b}\right) \geq \gamma C\left(\mathrm{SNR}_{l b}\right)$ holds for all $\mathrm{SNR}_{l b}$ of interest. In each of the three regimes of $(g, h, K)$, the powers $P_{1}$ and $P_{2}$ are chosen such that the value of $\mathrm{SNR}_{l b}$ is at most $\frac{1}{1+1+1 / K}$, which is contained in $[1 / 3,1 / 2]$. Hence, $\gamma$ can be chosen as

$$
\begin{aligned}
\gamma & =\inf _{1 / 3 \leq \mathrm{SNR}_{l b} \leq 1 / 2} \frac{C_{\text {binary }}\left(\mathrm{SNR}_{l b}\right)}{C\left(\mathrm{SNR}_{l b}\right)} \\
& =\frac{C_{\text {binary }}(1 / 2)}{C(1 / 2)} \\
& =\frac{0.29048}{0.29248} \\
& \geq 0.99,
\end{aligned}
$$

where the numerical values can be computed as described for example in [24, pp. 435-437].

Hence the rate per unit energy achieved by the proposed scheme is at least

$$
\frac{\gamma \mu \log (4 / 3)}{4} \cdot \min \{K g, \sqrt{K g h}, K h\} \geq \frac{\mu}{10} \min \{K g, \sqrt{K g h}, K h\} .
$$

This concludes the proof of Theorem 2 .

\section{Generalization to unequal drifts}

If the $k$ th IDC in the first hop has mean $\tilde{\mu}_{1, k}$ and the $k$ th IDC in the second hop has mean $\tilde{\mu}_{2, k}$, for $k \in\{1,2, \ldots, K\}$, then in place of (6), the $k$ th relay chooses a scaling factor $\alpha_{k}$ which is

$$
\alpha_{k}=\sqrt{\frac{P_{2 k}}{1+\tilde{\mu}_{1, k} g P_{1}}},
$$

where $P_{2 k}$ is the transmit power at the $k$ th relay. Instead of using a repetition code of the same length at all the relays, the length of the repetition codes used by the $k$ th relay is chosen to be $N_{k}^{\prime}$ such that $\tilde{\mu}_{2,1} N_{1}^{\prime}=\tilde{\mu}_{2,2} N_{2}^{\prime}=\ldots \tilde{\mu}_{2 K} N_{K}^{\prime}$, and the $N_{k}^{\prime}$ 's are sufficiently large, say $N_{1}^{\prime}=N^{4}$. Then, proceeding as in the previous subsection with a few minor changes, we end up with the following lower bound on the end-to-end SNR of the effective AWGN channel, a more general form than (23):

$$
\frac{\left(\sum_{k=1}^{K} \alpha_{k} \sqrt{\tilde{\mu}_{1, k} \tilde{\mu}_{2, k} g h}\right)^{2} P_{1}}{1+h \sum_{k=1}^{K} \tilde{\mu}_{2, k} \alpha_{k}^{2}} .
$$


Depending on the regime of $(g, h, K)$, we make the following choices, which yield after some simplification, the claim in (1).

- If $h<g / K$, we choose

$$
\begin{aligned}
P_{1} & =\frac{1}{\left(\min _{1 \leq k \leq K} \tilde{\mu}_{1, k}\right) g}, \\
P_{2 k} & =\frac{1}{\tilde{\mu}_{2, k} K^{2} h}, \quad 1 \leq k \leq K .
\end{aligned}
$$

- If $g / K \leq h<K g$, we choose

$$
\begin{aligned}
P_{1} & =\frac{1}{\left(\min _{1 \leq k \leq K} \tilde{\mu}_{1, k}\right) \sqrt{K g h}}, \\
P_{2 k} & =\frac{1}{\tilde{\mu}_{2, k} \sqrt{K^{3} g h}}, \quad 1 \leq k \leq K .
\end{aligned}
$$

- If $h \geq K g$, we choose

$$
\begin{aligned}
P_{1} & =\frac{1}{\left(\min _{1 \leq k \leq K} \tilde{\mu}_{1, k}\right) K g}, \\
P_{2 k} & =\frac{1}{\tilde{\mu}_{2, k} K h}, \quad 1 \leq k \leq K .
\end{aligned}
$$

\section{REFERENCES}

[1] Y.-C. Huang, U. Niesen, and P. Gupta, "Energy-efficient communication in the presence of synchronization errors," arXiv:1301.6589 [cs.IT], Jan. 2013, submitted to IEEE Trans. Inf. Theory.

[2] R. L. Dobrushin, "Shannon's theorems for channels with synchronization errors," Problems Inform. Transm., vol. 3, no. 4, pp. 11-26, 1967.

[3] M. Mitzenmacher, "A survey of results for deletion channels and related synchronization channels," Prob. Surv., vol. 6, pp. 1-33, 2009.

[4] Y. Kanoria and A. Montanari, "On the deletion channel with small deletion probability," in Proc. IEEE ISIT, June 2010, pp. $1002-1006$.

[5] A. Kalai, M. Mitzenmacher, and M. Sudan, "Tight asymptotic bounds for the deletion channel with small deletion probabilities," in Proc. IEEE ISIT, June 2010, pp. 997-1001.

[6] R. Venkataramanan, S. Tatikonda, and K. Ramchandran, "Achievable rates for channels with deletions and insertions," in Proc. IEEE ISIT, July 2011, pp. 346-350.

[7] M. Drmota, W. Szpankowski, and K. Viswanathan, "Mutual information for a deletion channel," in Proc. IEEE ISIT, July 2012, pp. 2561-2565.

[8] A. R. Iyengar, P. H. Siegel, and J. K. Wolf, "Modeling and information rates for synchronization error channels," in Proc. IEEE ISIT, July 2011, pp. 380-384.

[9] M. J. E. Golay, "Note on the theoretical efficiency of information reception using PPM," Proc. IRE, vol. 37, p. 1031 , Sept. 1949.

[10] S. Verdú, "On channel capacity per unit cost," IEEE Trans. Inf. Theory, vol. 36, no. 5, pp. 1019-1030, Sept. 1990.

[11] B. Schein and R. Gallager, "The Gaussian parallel relay network," in Proc. IEEE ISIT, June 2000, p. 22.

[12] B. Schein, "Distributed coordination in network information theory," Ph.D. dissertation, Massachusetts Institute of Technology, 2001.

[13] U. Niesen and S. Diggavi, "The approximate capacity of the Gaussian $N$-relay diamond network," IEEE Trans. Inf. Theory, vol. 59, no. 2, pp. 845-859, Feb. 2013.

[14] F. Parvaresh and R. Etkin, "Using superposition codebooks and partial decode-and-forward in low-SNR parallel relay networks," IEEE Trans. Inf. Theory, vol. 59, no. 3, pp. 1704-1723, Mar. 2013.

[15] A. El Gamal, M. Mohseni, and S. Zahedi, "Bounds on capacity and minimum energy-per-bit for AWGN relay channels," IEEE Trans. Inf. Theory, vol. 52, no. 4, pp. 1545-1561, Apr. 2006.

[16] A. S. Avestimehr and D. N. C. Tse, "Outage capacity of the fading relay channel in the low-SNR regime," IEEE Trans. Inf. Theory, vol. 53, no. 4, pp. 1401-1415, Apr. 2007.

[17] J. Massey, “Optimum frame synchronization,” IEEE Trans. Commun., vol. 20, no. 2, pp. 115-119, Apr. 1972.

[18] S. Verdú, "The capacity region of the symbol-asynchronous Gaussian multiple-access channel," IEEE Trans. Inf. Theory, vol. 35, no. 4, pp. 733-751, July 1989.

[19] T. Cover, R. McEliece, and E. Posner, "Asynchronous multiple-access channel capacity," IEEE Trans. Inf. Theory, vol. 27, no. 4, pp. 409-413, July 1981.

[20] J. Hui and P. Humblet, "The capacity region of the totally asynchronous multiple-access channel," IEEE Trans. Inf. Theory, vol. 31, no. 2, pp. 207-216, Mar. 1985.

[21] V. Chandar, A. Tchamkerten, and D. Tse, "Asynchronous capacity per unit cost," in Proc. IEEE ISIT, June 2010, pp. $280-284$.

[22] I. Shomorony, R. Etkin, F. Parvaresh, and S. Avestimehr, "Bounds on the minimum energy-per-bit for bursty traffic in diamond networks," in Proc. IEEE ISIT, July 2012, pp. 801-805. 
[23] R. Yeung, N. Cai, S.-W. Ho, and A. Wagner, "Reliable communication in the absence of a common clock," IEEE Trans. Inf. Theory, vol. 55, no. 2, pp. 700-712, Feb. 2009.

[24] J. G. Proakis, M. Salehi, and G. Bauch, Contemporary Communication Systems Using Matlab, 3rd ed. Cengage Learning, 2012. 